\title{
Well-being of Indians: A Lockdown Story
}

\author{
Vindhya Shetty $^{1}$, Manaswee Ruiwale ${ }^{1}$, Deepthi $\mathrm{R}^{1}$, and Deekshith D Shetty ${ }^{1}$ \\ ${ }^{1}$ Symbiosis Statistical Institute (SSI) Symbiosis International (Deemed) University
}

May 17, 2021

\begin{abstract}
A nationwide lockdown was initiated to prevent the worsening of the COVID-19 pandemic in India. This study aims to understand the impact of the lockdown on the well-being of Indians, mainly focusing on their life satisfaction and job satisfaction. Though, before the lockdown, family income was not related to life satisfaction, during the lockdown, it was found that higher income families experienced higher levels of life satisfaction. One's opinion on the beneficiality of the lockdown too was related to their level of income. Decline in both, job satisfaction and life satisfaction was seen. Gender, marital status and companions during the lockdown were significantly related to life satisfaction. Those who were married were more satisfied during the lockdown. Job satisfaction was irrespective of one's gender, marital status and educational qualification. An attempt has been made to understand the embracement of work from home culture by Indians. The study also tries to answer if the time one spends on oneself and on social media impact their well-being. A lot of changes were seen in the lifestyle and state of mind of people. With change in working conditions and income for a majority of people, there was uncertainty as to when things would normalize. The importance of companionship during these hard times was also revealed. Like every coin has two sides, this too has its positive side. People have inculcated habits that have improved their lifestyle. A lot of people have also found this as an opportunity to work on their relationships.
\end{abstract}

\section{Introduction}

India, a country with a population of around 1.38 billion went through a nationwide lockdown from 2403-2020 to 31-05-2020, which was then, based on the need of the hour, extended and reintroduced in many regions by the local authorities to fight the COVID-19 pandemic. This lockdown had a diverse impact on the country which has a very high social and economic inequality. Not only did it affect the economic condition of the citizens, but also their mental and physical state.

The legendary Greek philosopher Aristotle once said, "Man is by nature a social animal; an individual who is unsocial naturally and not accidentally is either beneath our notice or more than human. Society is something that precedes the individual". No man can lead a life as a loner, separated from society forever. He has to enter into relationships with fellowmen to truly live a life. Nobody can get away from this chain of mutual dependence. These clearly justify the importance of social life. This very idea of social interaction was cut down significantly. A major proportion of the population was confined within the four walls of their house with some companionship or none. Their interactions were restricted to only those they were residing with. There were instances of people having to stay away from their dear ones due the lockdown.

The life satisfaction of people might have been hampered due to the lockdown. The impact of the lockdown on the lives of people from various walks of life has been examined here. An attempt has been made to study the various causes for change in life and job satisfaction of people, i.e. to study the various parameters influencing life and job satisfaction, to what extent factors like gender, age, income level, employment status had an impact on the well-being of a person during the lockdown. 
The lockdown hit hard on the bellies of the people belonging to the daily wage categories, self-employed class, etc. There have been regular reports of how the economically backward class struggled to meet their daily needs. The study tests whether family income could be a key player in life satisfaction. Instances of employed people having lost their jobs during the lockdown gave us a scope to investigate their well-being in comparison to those who remained employed during the lockdown.

The lockdown had major changes on the working class. India was introduced to the concept of work from home on a very large scale. It also led to change in working hours and pay scale. Attempts have been to understand the changes taken place and the advantages of the work from home culture.

Job satisfaction is another important aspect of this study, which plays a vital role in the lives of the working class. It was interesting to study how various parameters affected job satisfaction. The effect of change in working hours on job satisfaction has also been studied.

Along with lockdown came the drastic change in the routine of people. People had leisure like never before. With this, came the scope of picking up new activities. People even had time to spend on themselves, called 'me time' in layman terms. In the present world, another important aspect of life is social media. It was interesting to study the change in the trend of time spent on social media before and during lockdown among the different sections of the society and its impact on life satisfaction.

The Indian society has always promoted family values. For a long time Indians lived under the joint family structure, but with modernization, the nuclear family structure has taken over. Family, being a significant part of the Indian society, is known to have a major impact on one's personal well-being in terms of productivity and satisfaction level. Taking this essence of life into consideration, the study strived to understand the relevance of family functioning, family involvement, mutual understanding and the impact of the lockdown on these factors.

Though a lot of pain and misfortune was witnessed during the lockdown, it had its brighter side too. There were various instances of people making the best use of these bad times and improving their daily life in terms of quality and performance. An attempt has also been made to understand how beneficial this lockdown was to people.

\section{Literature Review}

During this chaotic situation caused by the COVID-19 pandemic, where about half of the world's population has been under some form of lockdown, a lot of studies have taken place in this regard. One such study was carried out to investigate the impact of lockdown on the living habits of people and their social behaviour intending to measure the adjustments made by people and how they maintained their daily routine during the crisis (Mahendra Kumar \& Sachin Dwivedi, 2020). The study reflected the drastic changes in the lifestyle of people due to the lockdown with a lot of people adapting to work from home culture, installing new applications on their electronic devices, maximizing the use of internet data for official work and to access social media to improve communication facilities, thereby overcoming social isolation. People spent more time watching television, cooking, reading, sleeping and less time working out. The lockdown impacted the mental state of people as they were more worried about their family and friends. Forced to being socially isolated added to overthinking and anxiety. This lockdown also awakened the sense of belonging and solidarity among people as they provided mutual assistance towards their family and friends in motivating them to stay positive and passing on important information related to COVID-19. All in all, the study emphasized the need to monitor and gather research data to develop evidence-driven strategies to understand the adverse effects of the lockdown implementation and impacts caused by these unprecedented changes in people's daily lives, which is what this study tries to imbibe.

A salient aspect of an employed individual's well-being has been job satisfaction, and the attainment of such satisfaction during the lockdown seemed slightly skeptical. Khuntia and Adhikary ( 2015) emphasized on the different dimensions of job satisfaction and their impact over level of job satisfaction and level of 
performance of management college teachers in Orissa through statistical techniques like chi-square test of association and multiple regression analysis. No association between job satisfaction and performance was seen. Major determinants of job satisfaction included work pressure, salary and job security. It was also observed that the teachers had the fear of losing their jobs. Khuntia and Adhikary have restricted their study to academicians only. This study, however, has been an attempt to understand how the imposed lockdown has affected job satisfaction of the working class in general.

Similar studies have been carried out to understand the impact of different dimensions on life satisfaction which constitutes a major component of one's well-being. Toker (2012) assessed life satisfaction using Diener et al.'s Life Satisfaction Scale. Among the demographic variables, gender, age, income level, length of service in higher education, length of service at the present university and abroad experience were significantly related to life satisfaction, while marital status was not.

Edwards and Klemmack (1973) tested for partial effects and determined the extent to which each of the considered predictors make an independent contribution to explain perceived satisfaction. One of the major findings in their studies was that socioeconomic status, nonfamilial participation and health status were principal components in the predictive modelling of life satisfaction, socioeconomic status being the most important among them. They also concluded that people who were young, married and had larger families were more satisfied with life. The recent lockdown has brought about an ocean of changes in the daily lives of people, leading to a lot of changes in lifestyle. Through this study, an attempt has been made to validate the credibility of the previously done studies, specifically in the lockdown scenario. Comparison of satisfaction levels between the two phases, before and during the lockdown has been done.

Spatial differences in the impact of the Dutch lockdown was mapped on well-being and lifestyle in a study (Leeuwen \& Lepage, 2020). From initial analysis, they claimed that during the lockdown, on an average, well-being declined across the Netherlands. It differed significantly between rural and urban areas during the lockdown, but this was not the case before. The decline in well-being among urban dwellers was affiliated to their environment and population composition. Further, they put forth that the well-being of young people of age 18-25 deteriorated most due to the lockdown. Due to the vast cultural difference between Netherlands and India, the trend observed in life satisfaction in the two countries might vary a lot. This study is an attempt to understand these differences and similarities.

\section{Methodology}

\section{Respondents}

The data for the study was collected through a survey on Google Forms via various social media platforms like WhatsApp, Facebook and Linkedin. 385 was the required sample size considering a confidence level of $95 \%$, a $5 \%$ margin of error and the fact that $73.38 \%$ of the Indian population are above the age of 14 . From 1175 responses collected over a span of 13 days from 05-05-2020 to 17-05-2020 during the lockdown phase, only 1094 were taken into consideration after deletion of outliers and those responses that did not provide the necessary consent (44). The large sample size gives the hypothesis test a greater ability to detect small effects, thus having a higher statistical power. The respondents came from various walks of life. 
Table 1- Description of the Respondents

\begin{tabular}{|c|c|c|c|c|c|}
\hline Variable & & Female & Male & Total & Percentage \\
\hline \multirow[t]{5}{*}{ Age } & $15-23$ & 329 & 282 & 611 & 55.85 \\
\hline & $24-28$ & 81 & 87 & 168 & 15.36 \\
\hline & $29-35$ & 32 & 56 & 88 & 8.04 \\
\hline & $36-60$ & 68 & 134 & 202 & 18.46 \\
\hline & $60+$ & 6 & 19 & 25 & 2.29 \\
\hline \multirow[t]{3}{*}{ Locality } & Rural & 99 & 136 & 235 & 21.48 \\
\hline & Semi-urban & 111 & 164 & 275 & 25.14 \\
\hline & Urban & 306 & 278 & 584 & 53.38 \\
\hline \multirow[t]{2}{*}{ Marital Status } & Married & 124 & 190 & 314 & 28.70 \\
\hline & Single & 392 & 388 & 780 & 71.30 \\
\hline \multirow[t]{2}{*}{ Family Structure } & Joint Family & 117 & 151 & 268 & 24.50 \\
\hline & Nuclear Family & 399 & 427 & 826 & 75.50 \\
\hline \multirow[t]{6}{*}{ Educational Qualification } & Master's degree & 212 & 175 & 387 & 35.37 \\
\hline & Bachelor's degree & 280 & 336 & 616 & 56.31 \\
\hline & Diploma & 6 & 33 & 39 & 3.56 \\
\hline & Secondary(Std 8-12) & 15 & 33 & 48 & 4.39 \\
\hline & Primary(Std 1-7) & 2 & 0 & 2 & 0.18 \\
\hline & No schooling & 1 & 1 & 2 & 0.18 \\
\hline \multirow[t]{5}{*}{ Annual Family Income } & Less than 70,000 & 121 & 92 & 213 & 19.47 \\
\hline & $70,000-2,70,000$ & 149 & 161 & 310 & 28.34 \\
\hline & $2,70,000-8,45,000$ & 157 & 199 & 356 & 32.54 \\
\hline & $8,45,000-15,00,000$ & 63 & 78 & 141 & 12.89 \\
\hline & Greater than $15,00,000$ & 26 & 48 & 74 & 6.76 \\
\hline \multirow[t]{7}{*}{ Occupation } & Government job & 22 & 42 & 64 & 5.85 \\
\hline & Homemaker & 41 & 0 & 41 & 3.75 \\
\hline & Private job & 115 & 168 & 283 & 25.87 \\
\hline & Retired & 6 & 22 & 28 & 2.56 \\
\hline & Self-employed & 12 & 74 & 86 & 7.86 \\
\hline & Student & 309 & 253 & 562 & 51.37 \\
\hline & Unemployed & 11 & 19 & 30 & 2.74 \\
\hline Total & & 516 & 578 & 1094 & \\
\hline
\end{tabular}

\section{Methods}

- Data visualization is a very important aspect in the field of data analytics. The human brain processes visual information quicker and more efficiently than mere numbers or reports, and so, a few visualizations have been used here.

- A Likert Scale is an orderly scale from which respondents choose the option that best supports their opinion, and has been used to measure life satisfaction, job satisfaction and beneficiality of the lockdown as it helps in capturing the intensity of the respondents' feeling towards the factor.

- The Kruskal-Wallis test has been extensively used in the study for analyzing the relationship between various parameters. It is a non-parametric test used for comparing two or more independent samples 
with null hypothesis being the equality of medians of all groups. This test was opted keeping in mind the non-normality of the data, thus giving Kruskal-Wallis test an edge over the parametric alternative of ANOVA. Once the Kruskal-Wallis test has been applied, the next step would be to identify the particular pairs of samples that have medians significantly different from each other. Here, Dunn's Post Hoc test comes handy. It is one of the non parametric alternatives to Tuckey's Post Hoc test. The null hypothesis under Dunn's test is the absence of significant difference between the medians of the groups.

- In the study, Mann-Whitney U test has also been used, which is a non-parametric test of the null hypothesis that the probability that a randomly selected value from one population is less than a randomly selected value from a second population is equal to the probability of being greater. It is the non-parametric alternative to independent samples t-test.

The Kruskal-Wallis, Dunn's Post Hoc test and Mann-Whitney U and have been applied to study the relationships between various parameters such as occupation, age, income level, marital status, family structure, life satisfaction, job satisfaction, beneficiality of lockdown, time spent on social media and time spent on oneself.

- Wilcoxon Signed-Rank test is a non-parametric statistical hypothesis test used to compare two related samples, matched samples, or repeated measurements on a single sample to assess whether their population mean ranks differ. It is the non-parametric alternative to Paired Student's t-test. The test is applicable to non-normal data, which is the case here. It has been used to study the impact of lockdown on time spent on oneself and social media, job satisfaction and life satisfaction.

- The term Level of Significance, denoted by $\alpha$, is the probability of rejecting the null hypothesis when it is true. It shows how likely a pattern in the data is due to chance and is determined before conducting the experiment. In this study, all statistical tests were carried out at $5 \%$ Level of Significance.

- It was seen that categories of some parameters consisted of a very small number of data points. Such data points were excluded from further analysis. These included:

1 respondent opting gender as 'Other'

1 Divorcee and 3 Widowed respondents

23 respondents not currently residing in India

3 respondents of age less than 15

6 respondents having reported unrealistic hours spent on social media and oneself

- The parameters 'Time spent on social media' and 'Time spent on oneself' were converted into class intervals for the convenience of analysis. The intervals were-'Less than 1', '1-3', '3-5' and 'More than 5' (in hours) .

- The first three groups under Educational Qualification were pooled during analysis as the sample size was low.

- All the variables were included in the analysis.

- Data and codes have not been made available for access. Analyses were performed using the open source programming language Python.

\section{Limitations}

- The data was collected online, since conducting a field survey was difficult, given the situations due to COVID-19. Thus, there is a definite bias in the data as only those with access to smartphones and internet connection have been a part of the survey. People from remote areas might not have been properly represented in the study.

- If an individual or their close ones have been tested COVID-19 positive could be a factor that might bring about interesting results. 
- Geographic location of an individual has not been considered as a factor. Based on whether or not the location is a highly/mildly COVID-19 infected zone, a significant change in the well-being of individuals might show up.

- Whether somebody is closely dealing with COVID-19 patients / quarantined people at the workplace could be another factor that could bring about interesting insights.

\section{Results and Discussion}

\begin{tabular}{|c|c|c|c|c|c|c|c|c|c|}
\hline \multicolumn{10}{|c|}{ Table 2- Results } \\
\hline & Factor & Comparison & Statistic & p-value & & Factor & Comparison & Statistic & p-value \\
\hline Life Satisfaction & & & & & ||Beneficiality & & & & \\
\hline \multirow[t]{8}{*}{ Before } & $\mathrm{Age}$ & & $7.344(\mathrm{KW})$ & 0.119 & & $\mathrm{Age}$ & & $2.646(\mathrm{KW})$ & 0.619 \\
\hline & Gender & Malc $>$ Fcmalc & $1168910.5(\mathrm{MW})$ & 0.000 & & Gender & & $0.018(\mathrm{KW})$ & 0.893 \\
\hline & Locality & & $0.786(\mathrm{KW})$ & 0.675 & & Locality & & $2.972(\mathrm{KW})$ & 0.226 \\
\hline & Marital Status & & $116661(\mathrm{MW})$ & 0.099 & & Marital Status & & $2.032(\mathrm{KW})$ & 0.154 \\
\hline & Family Structure & & $3.486(\mathrm{KW})$ & 0.062 & & Family Structure & & $0.259(\mathrm{KW})$ & 0.611 \\
\hline & Education & & $12.206(\mathrm{KW})$ & 0.032 & & Companions & & $3.654(\mathrm{KW})$ & 0.161 \\
\hline & Annual Income & & $1.559(\mathrm{KW})$ & 0.816 & & Education & & $6.861(\mathrm{KW})$ & 0.231 \\
\hline & Occupation & & $7.713(\mathrm{KW})$ & 0.260 & & Annual Income & & $14.003(\mathrm{KW})$ & 0.007 \\
\hline \multirow[t]{21}{*}{ During } & Age & & $6.864(\mathrm{KW})$ & 0.143 & & Occupation & & $7.268(\mathrm{KW})$ & 0.297 \\
\hline & Gender & & $0.471(\mathrm{KW})$ & 0.492 & & Current Working Status & & $2.521(\mathrm{KW})$ & 0.283 \\
\hline & Locality & & $5.123(\mathrm{KW})$ & 0.077 & & Working Hours for WFH & Reduced $\leqslant$ No change & $2966(\mathrm{MW})$ & 0.007 \\
\hline & Marital Status & Married $>$ Single & $109489(\mathrm{KW})$ & 0.002 & & & Reduced $\backslash$ Increased & $2818.5(\mathrm{MW})$ & 0.002 \\
\hline & Family Structure & & $0.294(\mathrm{KW})$ & 0.588 & & Mutual Understanding & & $25.272(\mathrm{KW})$ & 0.000 \\
\hline & Companionship & Alone $<$ Family & $9367(\mathrm{KW})$ & 0.017 & & & Increased $>$ No clange & $110870.5(\mathrm{MW})$ & 0.000 \\
\hline & Education & & $18.03(\mathrm{KW})$ & 0.000 & & Family Functioning & & $73.296(\mathrm{KW})$ & 0.000 \\
\hline & Annual Income & & $25.173(\mathrm{KW})$ & 0.000 & & & Improved $>$ No change & e $87546.5(\mathrm{MW})$ & 0.000 \\
\hline & Occupation & & $11.164(\mathrm{KW})$ & 0.083 & & & Improved $>$ Worsened & $27666.5(\mathrm{MW})$ & 0.001 \\
\hline & Current Working Status & & $16.65(\mathrm{KW})$ & 0.000 & & Involvement with Family & & $22.304(\mathrm{KW})$ & 0.000 \\
\hline & & Not Working $<$ WFH & $14847.5(\mathrm{MW})$ & 0.000 & & & Increased $>$ No clange & $=100055(\mathrm{MW})$ & 0.000 \\
\hline & & Not Working $<$ Work from workplace & e 4924(MW) & 0.008 & |Self Time & & & & \\
\hline & Working Hours for WFH & Reduced $<$ No change & $3068(\mathrm{MW})$ & 0.017 & |Bcfore & $\mathrm{Agc}$ & & $12.97(\mathrm{KW})$ & 0.011 \\
\hline & Mutual Understanding & & $13.479(\mathrm{KW})$ & 0.001 & & Marital Status & Married < Single & $141325(\mathrm{MW})$ & 0.000 \\
\hline & & Increased $\gg$ No clange & $116969.5(\mathrm{MW})$ & 0.000 & & Family Structure & Nuclear>Joint & $118572.5(\mathrm{MW})$ & 0.035 \\
\hline & Family Functioning & & $83.507(\mathrm{KW})$ & 0.000 & & Lifc Satisfaction & & $7.284(\mathrm{KW})$ & 0.063 \\
\hline & & Improved $>$ No change & $86156(\mathrm{MW})$ & 0.000 & |During & Age & & $21.925(\mathrm{KW})$ & 0.000 \\
\hline & & Improved $>$ Worsened & $14850(\mathrm{MW})$ & 0.000 & & Marital Status & Married $<$ Single & $144799(\mathrm{MW})$ & 0.000 \\
\hline & & Same>Worsened & $26947.5(\mathrm{MW})$ & 0.000 & & Family Structure & Nuclear $>$ Joint & $119487.5(\mathrm{MW})$ & 0.024 \\
\hline & Involvement with Family & Increasse $\gg$ No clange & $110197(\mathrm{MW})$ & 0.008 & & Life Satisfaction & & $4.638(\mathrm{KW})$ & 0.200 \\
\hline & Lockdown Impact & During $<$ Before & $116510.5(\mathrm{WSR})$ & 0.013 & & Lockdown Impact & During $>$ Before & 33593.5 (WSR) & 0.000 \\
\hline \multirow{6}{*}{$\begin{array}{l}\text { Job Satisfaction } \\
\text { Before }\end{array}$} & & & & & | Social Media Tim & & & & \\
\hline & Gender & & $21661.5(\mathrm{KW})$ & 0.335 & |Before & Age & & $127.994(\mathrm{KW})$ & 0.000 \\
\hline & Locality & & $1.672(\mathrm{KW})$ & 0.433 & & & & & \\
\hline & Marital Status & & $1.501(\mathrm{KW})$ & 0.220 & & Marital Status & Married $<$ Single & 175009 (MW) & 0,000 \\
\hline & Education & & $8.872(\mathrm{KW})$ & 0.114 & & Family Structure & Nuclear>Joint & 87104(MW) & 0.000 \\
\hline & Annual Income & & $3.935(\mathrm{KW})$ & 0.415 & & & & & \\
\hline \multirow[t]{9}{*}{ During } & Gender & & 13371(KW) & 0.471 & & Lifc Satisfaction & & $2.057(\mathrm{KW})$ & 0.561 \\
\hline & Locality & & $0.437(\mathrm{KW})$ & 0.804 & |During & Agc & & $146.663(\mathrm{KW})$ & 0.000 \\
\hline & Marital Status & & $2,443(\mathrm{KW})$ & 0.118 & & Marital Status & Married $<$ Single & $177128.5(\mathrm{MW})$ & 0.000 \\
\hline & Education & & $7.889(\mathrm{KW})$ & 0.162 & & Family Structure & Nuclear $>$ Joint & 78352(MW) & 0.000 \\
\hline & Annual Income & & $6.032(\mathrm{KW})$ & 0.197 & & Lifc Satisfaction & & $18.021(\mathrm{KW})$ & 0.000 \\
\hline & Working Hours for WFH & Reduced $<$ No clange & $2170(\mathrm{MW})$ & 0.000 & & Lockdown Impact & During $>$ Before & 21581(WSR) & 0.000 \\
\hline & & Reduced < Increased & $2777.5(\mathrm{MW})$ & 0.001 & & & & & \\
\hline & Salary for Not working & Paid like usual>Not paid & $704(\mathrm{MW})$ & 0.046 & & & & & \\
\hline & Lockdown Impact & During $<$ Before & 19801(WSR) & 0.000 & & & & & \\
\hline
\end{tabular}

Age, gender, locality, marital status, family structure, occupation and whether an individual is a working professional or a student were not related to the levels of life satisfaction of an individual. But during the lockdown, it was found that married people were more satisfied. An earlier study had suggested that young, married and those with large families were more satisfied (Edwards \& Klemmack, 1973). Also, people who lived alone through the phase of lockdown, probably because they were stranded away from home, had lower levels of life satisfaction than the ones spending the lockdown with their families. A person's level of education was related to life satisfaction, both, before and during the lockdown phase. Interestingly, annual family income was related to life satisfaction only during the lockdown. On further application of tests, it was seen that people with higher family incomes were more satisfied. The lockdown had put in a lot of problems for people to face, where there was less or no income as daily activities had been highly hampered. Those with lower income might not have had huge savings to live on satisfactorily and might have been very anxious as expenditures on food and shelter remained during the lockdown even when there was no stable income due to loss of work. This shows that in normal times, earning less money simply doesn't imply lower levels of satisfaction, and income is not really an important dimension affecting one's level of life satisfaction, but in times of an emergency like this pandemic has brought, the case is otherwise. Contradictions were seen as age, gender and income were considered significant in an earlier study (Toker, 2012). People who worked 
from home but had decreased working hours maybe because working from home doesn't ideally support their work type, were less satisfied. Uncertainty if this work from home culture would let them retain their jobs for long might have been the reason. Change in working hours had not affected satisfaction of those working from the workplace. Those who had not been able to work at all because of the lockdown were the least satisfied.

Fig. 1- Current Work Status

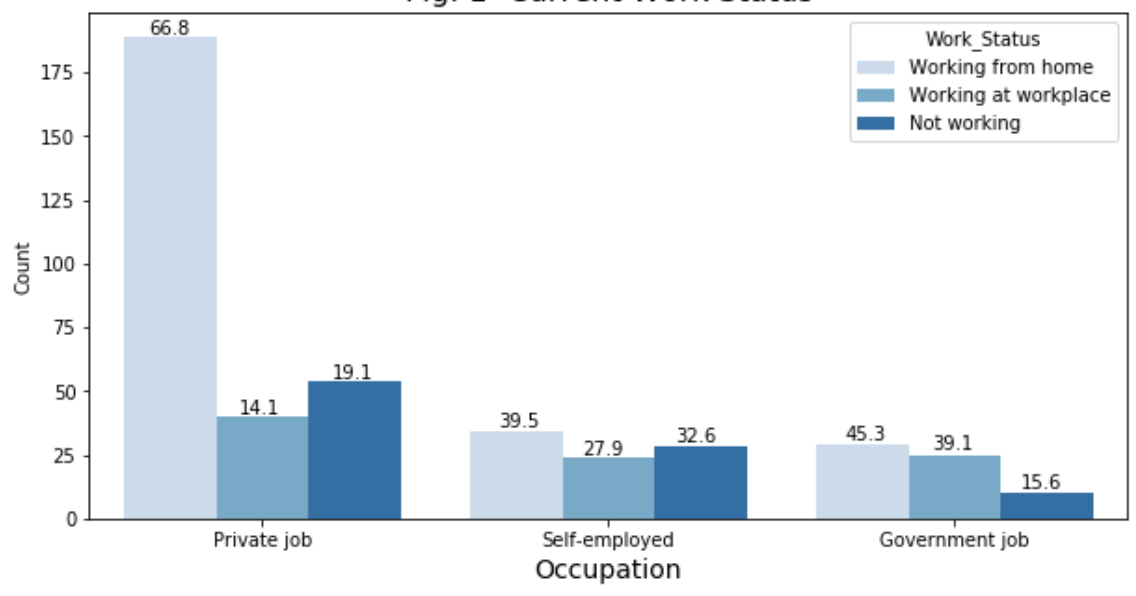

Beneficiality of the lockdown was irrespective of most factors like age, gender, locality, education, marital status, family structure, companions during the lockdown, occupation, current working status and whether an individual is a working professional or a student. Income however, was a significant factor. Those with the least income level, i.e., less than Rs 70000, felt the lockdown was least beneficial. Like life satisfaction, beneficiality of the lockdown too, was less for those working from home but, with decreased working hours. Change in working hours had not affected the beneficiality of those working from the workplace.

Job satisfaction was irrespective of gender, marital status, education level and locality. Differences were seen in the well-being of the rural and the urban in Netherlands (Leeuwen \& Lepage, 2020), which was not the case here. Contrary to the common belief, level of income was not related to job satisfaction levels. Like life satisfaction, job satisfaction too was less for those working from home and had decreased working hours. Change in working hours had not affected job satisfaction of those working from the workplace. Among the people who actually belonged to the workforce, but had not been able to work during the lockdown, either from home or workplace, those who were getting paid as usual had higher levels of life satisfaction than those who were getting paid unusually less or not getting paid at all.

Giving some time to oneself is a great practice that helps in reducing stress by doing something that one likes and benefits that person. It was seen on further tests that younger people could make more time for themselves, both before and during the lockdown phase. Married people could not make as much time for themselves as those who were single. Also, people from joint families were able to give less time to themselves than those from nuclear families before and during the lockdown. Contrary to what was expected, level of life satisfaction was not much affected by the time people gave themselves.

Age of an individual was affecting the amount of time they spent on social media platforms. Like time to oneself, younger people could afford to spend more time on social media than the older. It was seen that those of age group 15-23 spent more time than everybody else. People who were single, comparatively spent more time on social media. In joint families, the presence of a lot of family members, probably keeps individuals more engaged and leaves them with less time to spend on social media than the ones from nuclear families. It was seen that during the lockdown, those who spent more than five hours on social media had lower levels of life satisfaction. The reason could be the presence of a lot of negativity and fake information 
over these platforms that can only make a person feel anxious and uncertain about everything.

Those with increased mutual understanding amongst the members of their family found the lockdown to be more beneficial and had higher levels of life satisfaction. But, on further tests, it was seen that these results were not applicable to joint families. Those who thought their family functioning had improved during the lockdown had higher levels of life satisfaction and also found the lockdown more beneficial. Those who claimed that their involvement with family members had increased during the lockdown had considered the lockdown more beneficial and also had higher levels of life satisfaction. Nuclear families showed the same results, while no relationship between involvement with family and beneficiality or life satisfaction during the lockdown was found in joint families. Apart from the negative impact the confinement has brought to people, it was seen that people have seen this as an opportunity to work on their relationships with their families, and thus found the lockdown to be beneficial.
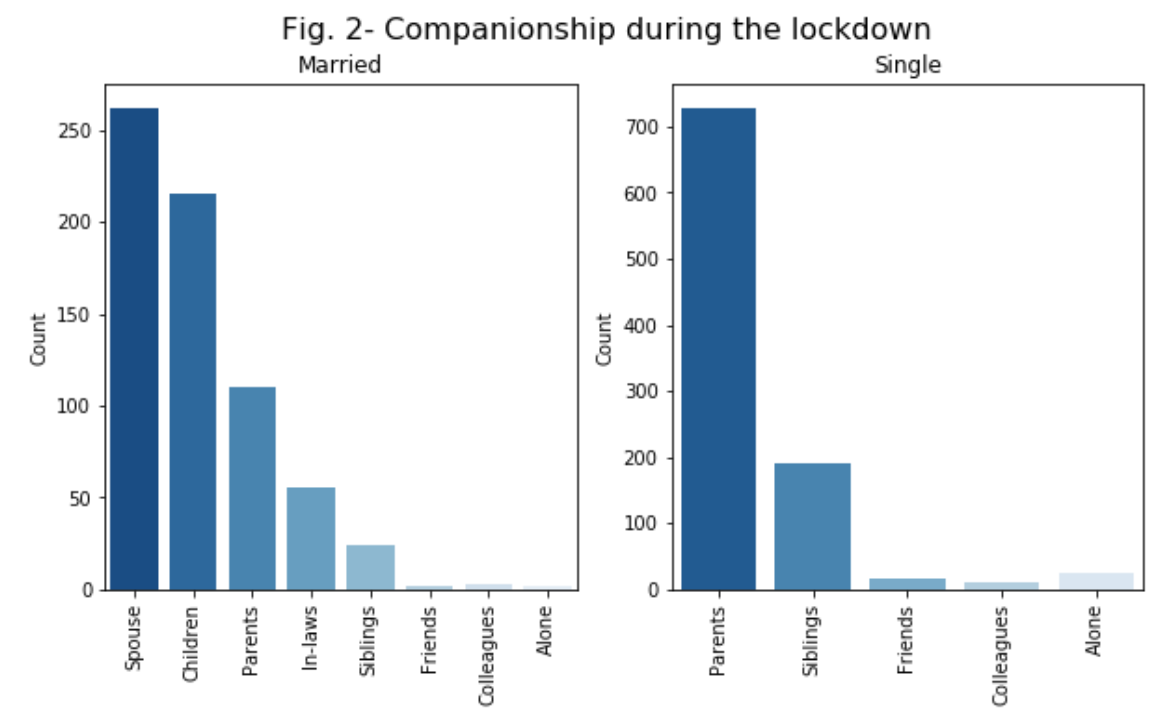

As was expected, people had more leisure time in hand, as daily activities had been immensely hampered, working hours were mostly reduced and classes for students stood suspended. People were thus able to give more time to themselves during the lockdown doing something they loved and benefited them. A lot of people reported having taken up new activities, something they probably never found time to do amidst their daily schedule. Fig. 3, consists of percentages of people taking up a few common activities before and during the lockdown.

A lot of people have made positive changes in their lifestyle by making meditation (7\%) and exercise (10.9\%) a part of their daily routine. The increase $(18.2 \%)$ in cooking could be mainly because of the absence of home helpers or cooks, which has pushed people into cooking for themselves and their families. The abundance of time might have encouraged a lot of people to learn and experiment with cooking. Be it students or working professionals, they generally do not have time left in their hectic schedules to indulge in online courses. The work from home and study from home culture might have saved them time to take up courses online (16\%). During the lockdown, more people have taken up leisure activities like watching movies and series, reading, writing, playing and art. Time spent on social media also has gradually improved. This was mostly in agreement with a previous study, except that, more people had begun working out (Kumar \& Dwivedi, 2020). 
Fig. 3- Activities taken up

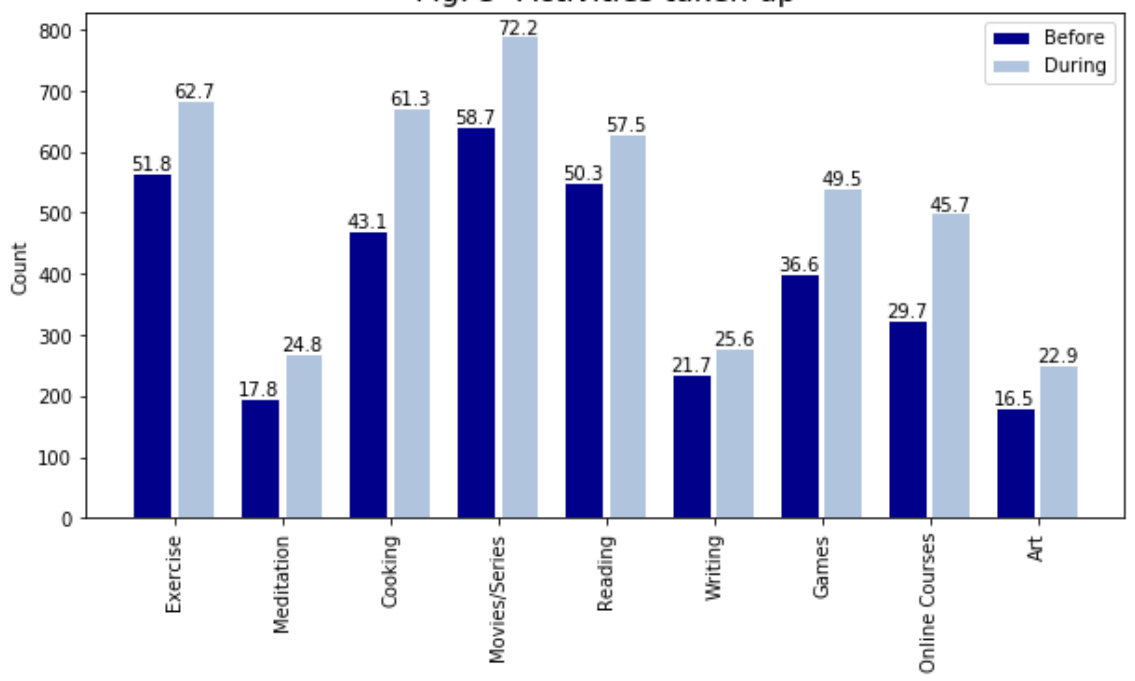

The life satisfaction of individuals had, as expected, declined during the lockdown phase, as was indicated by a prior study (Leeuwen \& Lepage, 2020). When tried to understand their state of mind during the lockdown, it was seen that $61.2 \%$ felt they were in a normal state of mind and $31.1 \%$ were happy. $19.1 \%$ people said they were worried, $7.7 \%$ unhappy, $6 \%$ depressed and $4 \%$ scared. People from places that had a high number of COVID-19 cases could be the ones who were worried and scared. People who had been stuck all alone during the lockdown, not being able to go home due to lack of transportation facilities, or to eliminate the risk of institutional quarantine could be the ones who felt lonely and depressed.

Job satisfaction too shows a gradual decline during the lockdown. This was in line with what was expected, as a lot of people have not been able to work at all because of the imposed conditions. Those who have been working from their workplaces have mostly been associated with essential services, and working may have been stressful given the situation and fear of getting the disease from their workplace. $62.7 \%$ of the people who have been working from home have found the work from home culture beneficial and have accepted it positively. Table 3 shows how the culture was beneficial to them. The most popular reasons were flexible schedules and the availability of homely and timely food. This also gave them a great opportunity to spend time with their loved ones. Many also suggested they could now give better time to their children even on a working day. Some thought that having their home as their workplace made them more comfortable. Not having to commute to their workplace saved people a great deal of time, especially in major cities with heavy traffic. Even with working hours remaining intact, the time saved from commuting could have helped them spend it on things that were way more productive. Working professionals, in general, do not have time left in their busy schedules to indulge in learning something new. This lockdown has provided them with ample time to indulge in online courses and other activities.

\section{Table 3- WFH Benefits}

\begin{tabular}{ll}
\hline Zero commuting & $31.70 \%$ \\
Homely Food & $40.90 \%$ \\
Comfortable environment & $34.90 \%$ \\
More time with loved ones & $36.50 \%$ \\
Flexible schedule & $41.30 \%$ \\
Saves money & $38.50 \%$ \\
\hline
\end{tabular}




\section{Conclusion}

Having been confined to stay indoors for a very long period due to the imposed lockdown in an attempt to curb the worsening of the COVID-19 situation has greatly impacted people's well-being. The fact that people cannot move around freely and socialize with people in a non-virtual way has also affected them. Lifestyle and state of mind has changed significantly. There is uncertainty in the minds of people as to when things would normalize. People are very much hopeful about a vaccine being discovered soon, so that the situation would not prolong for a long time. A number of sociodemographic, psychological and economic factors were included in the study that have helped to understand in what way they were related to the overall well-being of an individual. Having seen that during pandemics like this, income was significantly affecting well-being, the government could focus more on the welfare of the poor whose work has been greatly hampered. The study also showed the importance of having one's family around, and so, in any outbreaks to come in future, special care could be taken in helping people reach their families before the imposition of restrictions like this. Apart from the lockdown having impacted individuals negatively, there has been a bright side too. People have finally found the time to do things they have always wanted to do, learn things they never were able to. People were forced to stay indoors with the same faces everyday. Most of those who stayed with their families, or even those who have stayed away from home, have found this period as an opportunity to work on and improve their relationships, in person, or through virtual conversations. There are certain limitations in the study which opens up a lot of scope for further research.

\section{Declarations}

\section{Funding}

The study has not been granted any funds.

\section{Conflicts of Interest}

The authors declare that there is no conflict of interest.

\section{Acknowledgment}

We thank Mr Jairam Kanna, student, M.Sc Applied Statistics for his assistance in designing the survey and collection of data. We are grateful to all those who have taken time to respond to our survey, and our families and friends who have helped us reach more people. We also express our gratitude to those who have

been a part of the pilot survey, made recommendations and helped us improve it further. We also thank Symbiosis Statistical Institute for their support during the course of this research.

\section{Funding}

The study has not been granted any funds.

\section{Conflicts of Interest}

The authors declare that there is no conflict of interest.

\section{References}

Singh A, Singh G, Zaidi S.Z.H \& Dandona A. (2020). A study on emotional well-being midst coronavirus pandemic lockdown, Mukt Shabd Journal, Volume IX, Issue V, May/2020 
Banjare P, Dwivedi R \& Pradhan J. (2015). Factors associated with the life satisfaction amongst the rural elderly in Odisha, India,Health Qual Life Outcomes 13, 201 (2015) .https://doi.org/10.1186/s12955-0150398-y

Bhat BA, Khan S, Manzoor S et.al. (2020). A study on impact of COVID-19 lockdown on psychological health, economy and social life of people in Kashmir, International Journal of Science $\& 3$ Healthcare Research. 2020; 5(2): $36-46$

Toker B (2012). Life satisfaction among academicians: an empirical study on the universities of Turkey, Procedia - Social and Behavioral Sciences, 47 ( 2012) 190 - 195, CY-ICER 2012

Leeuwen E, Lepage L (2020). Spatial differences and the impact of the lockdown on well-being in the Netherlands.http://dx.doi.org/10.2139/ssrn.3597707

Edwards J, Klemmack D (1973). Correlates of Life Satisfaction: A Re-examination, Journal of Gerontology 1973, Vol. 28, No. 4, 497-502

Kumar M, Dwivedi S (2020). Impact of Coronavirus Imposed Lockdown on Indian Population and their Habits, International Journal of Science and Healthcare Research, Vol.5, Issue: 2

Miglani A (2020). Effect of lockdown during COVID-19: an Indian perspective, International Journal of Science \&3 Healthcare Research, 2020; 5(3): 55-61

Khuntia S, Adhikary S (2015). A Study on Job Satisfaction and Performance of Management Guru's of Management Colleges-with Reference to Odisha, International Journal of Research and Development - A Management Review (IJRDMR), Vol.4, Issue:1

Ehly S and Reimers T (1986). Perceptions of job satisfaction, job stability, and quality of professional life among rural and urban school psychologists, Psychology in the Schools, Volume 23, April 1986 